\title{
TEMPERATURE FLUCTUATIONS OF THE MICROWAVE BACKGROUND IN \\ PRIMEVAL ISOCURVATURE BARYON MODELS
}

\author{
S. N. DUTTA AND G. EFSTATHIOU \\ Department of Physics \\ University of Oxford \\ OX1 3NP, United Kingdom
}

\begin{abstract}
We calculate the temperature fluctuations in the microwave background in open primeval isocurvature baryon models (Peebles, 1987) with cosmological densities in the range $0.05 \leq \Omega \leq 0.2$ We assume that the power spectrum of fluctuations is a power law with the index varying between $-1 \leq n \leq 0$, as indicated by observations of large scale structure in the Universe. The Universe is assumed to be always fully ionized. The South Pole 13 field point experiment (Schuster et al., 1993) is compared to our theoretical predictions, and we find that the models predict larger temperature fluctuations than are observed. The observed temperature fluctuations on intermediate scales of $\lesssim 1^{\circ}$ thus seem difficult to reconcile with the isocurvature baryon model.
\end{abstract}

\section{Introduction}

The Cosmic Background Radiation (CBR) remains, one of the most powerful diagnostics of the Universe on large scales. The discovery of the temperature anisotropies in the CBR at various angular scales allows us to place new constraints on the formation of structure in the Universe. One of the models of structure formation that has been around for a long time is the Primeval Isocurvature Baryon (PIB) model (Peebles, 1987). In this model, the universe is open $(\Omega \lesssim 0.2)$ and is uniform at early times except for small fluctuations in the entropy per baryon. The main attraction of the picture is, of course, that there is no need for exotic dark matter (Peebles, 1987). 
Most dynamical measures of $\Omega$ indicate a value around 0.2 , suggesting the Universe is open. The limit on $\Omega_{b}$ from standard Big Bang nucleosynthesis is significantly less at $0.0125 h^{-2}$ (Walker et al., 1991), where $h$ is the Hubble's constant in units of $100 \mathrm{~km} \mathrm{~s}^{-1} \mathrm{Mpc}^{-1}$. With a low $h$ it is may be possible to explain the light element abundance via inhomogeneous nucleosynthesis with a value $\Omega_{b}$ as high as 0.1 (Gnedin \& Ostriker, 1992), overlapping with the lower range of $\Omega$ determined from dynamical studies (eg. Davies \& Peebles, 1982). Further, new observations of microlensing of the stars in the Large Magellanic Cloud (Udalski et al., 1994) indicate the possibility of the existence of a large population of "dark" compact objects. For these reasons, there has been some renewed interest in the PIB model (Cen et al., 1993).

One inelegant aspect of the PIB model is that, as yet, there is no compelling theory for the origin of the entropy fluctuations. The power spectrum of the initial entropy fluctuations is therefore not fixed, and can be varied "by hand" to fit observations. This arbitrariness makes testing of the model difficult. One way around the problem is to use the observational constraints on the power spectrum on scales at which the temperature fluctuations are measured. This is the approach we adopt in the present work.

The PIB models are distinguished by the early formation of structure, which can produce enough ionizing radiation to reionize the universe at early times. Previous work (eg. Efstathiou \& Bond, 1987) has shown that reionization is required in PIB models to satisfy the constraints on temperature anisotropies on scales of a few arcminutes. We therefore assume that the Universe is always ionized and compute the temperature anisotropies on intermediate angular scales.

\section{The Temperature Fluctuations}

\subsection{THE POWER SPECTRUM}

The temperature fluctuation of the CMBR in a PIB model is a combination of the initial entropy fluctuations, a small Sachs-Wolfe effect and a large contribution from Thomson scattering off moving electrons. We will consider angular scales where the Sunyaev-Zeldovich (Zeldovich \& Sunyaev, 1970 ) and second order effects (Vishniac, 1987, Efstathiou, 1988) are utterly negligible. The standard Boltzmann equations (Bond \& Efstathiou, 1987) for the perturbed distribution functions for the radiation are solved by expanding the relative temperature fluctuations $(\Delta T / T)$ in terms of Legendre polynomials. We assume the universe to be spatially flat in calculating the connection between the angular scale and the different orders of the polynomial expansion. This should be accurate at the angular scales we are interested in. 


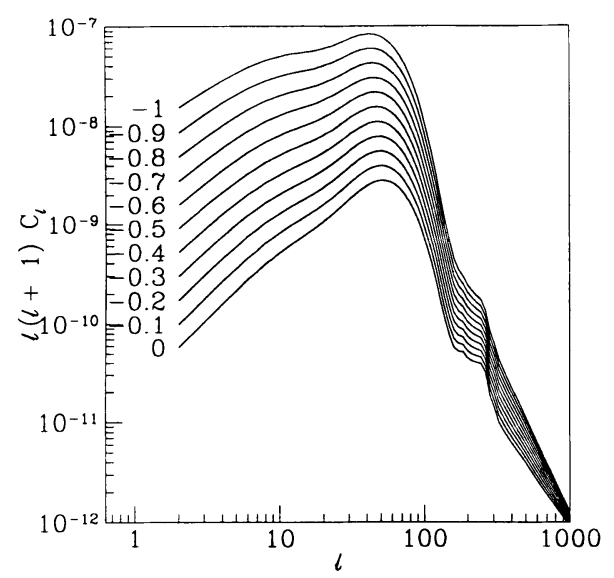

Figure 1. The power spectrum of the temperature fluctuations of the CMBR. $\Omega_{b}=0.1$ and $h=0.5$. The entropy fluctuations have been normalised to $\sigma_{8}=1$. The numbers next to each line give the spectral index of the initial entropy perturbations.

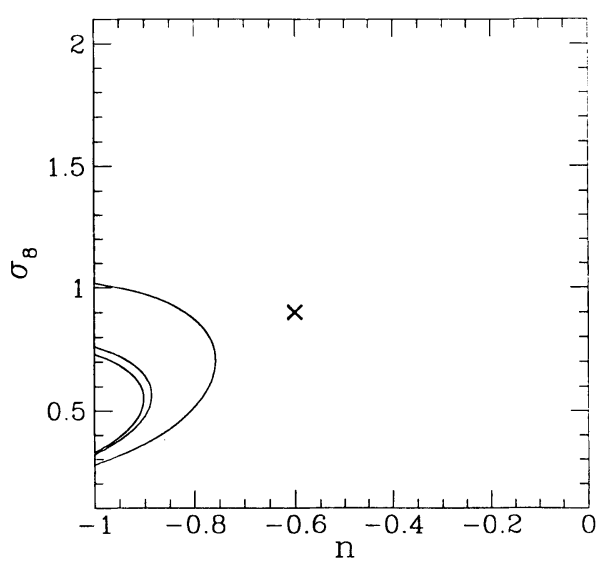

Figure 2. The contours of constant likelihood in the $n-\sigma_{8}$ plane in the Universe with $\Omega_{b}=0.1$ and $h=0.5$. The contours are the confidence levels with $99 \%, 98 \%$ and $68 \%$ probability. The cross indicates the best values from observations of large scale structure.

The power spectrum of the CBR temperature fluctuations is given in terms of the coefficients of the expansion as,

$$
C_{l}=\frac{V_{x}}{8 \pi} \int_{0}^{\infty} k^{2} d k\left|\Delta_{T l}\left(k, \tau_{0}\right)\right|^{2}
$$

As mentioned before this needs to be normalised to some initial value of the entropy fluctuations. We normalise the spectrum such that the r.m.s. fluctuations in the mass distribution in spheres of radius $8 h^{-1} \mathrm{Mpc}, \sigma_{8}$, is unity. The power spectrum of the temperature fluctuations for $\Omega_{b}=0.1$ and $h=0.5$ is shown in Fig. 1, for various values of the primordial spectral index, $n$.

\subsection{COMPARISON WITH OBSERVATIONS}

We have compared the models with the temperature anisotropies observed in the South Pole experiment of Schuster et al., (1993). This was a balloon borne and ground based experiment that used a cryogenic HEMT amplifier on a $1 \mathrm{~m}$ telescope. Measurements were made on four bands of equal ( 2.5 $\mathrm{GHz}$ ) width. Several scans were made, each of which consisted of stepping between 15 target points on the same elevation, seperated by $2^{\circ} .1$. The full width at half-maximum was 1.5 . 
We perform a likelihood analysis, assuming the fluctuations follow a Gaussian distribution. For each model we maximise the likelihood function,

$$
L \propto \frac{1}{\sigma_{8}^{N}\left|C_{i j}\right|^{1 / 2}} \exp \left\{-\frac{\sum x_{i} x_{j} C_{i j}^{-1}}{2 \sigma_{8}^{2}}\right\}
$$

with respect to the parameter $\sigma_{8}$. For the SP13 experiment, the covariance matrix $C_{i j}^{\prime}$ is given by ,

$$
C_{i j}=2 \sum_{l} C_{l} F_{l}^{2} \sum_{m>0} Y_{l}^{|m|}\left(\cos \theta_{i}, 0\right) Y_{l}^{|m|}\left(\cos \theta_{j}, 0\right) \cos \left[m\left(\phi_{i}-\phi_{j}\right)\right] H_{0}^{2}\left(m \phi^{*}\right)
$$

where $F_{l}=\exp \left[-l^{2} \theta_{s}^{2} / 2\right]$ and $H_{0}$ are the Struve functions and $\theta_{s}$ is the beam resolution.

Fig. 2 shows the likelihood contours in the $\sigma_{8}-n$ plane for the PIB model with $\Omega_{b}=0.1$ and $h=0.5$. The temperature fluctuation of the microwave background favours low $\sigma_{8}$ around 0.6 and $n$ around -1 . However, observations of large scale structure (Cen et al., 1993) favour $\sigma_{8} \simeq 0.9$ and $n \simeq-0.6$, (indicated by a cross in Fig. 2), so it appears that the model fails to match the observations. We find similar results for models with $\Omega_{b}=0.2$ and 0.05 and $h=0.5$.

The SP13 experiment thus sets strong constraints on the PIB model. We are currently investigating a wider range of model parameters and comparisons with other experiments on intermidiat angular scales of $1^{\circ}$. However, this analysis does indicate that anisotropy experiments on intermidate angular scales can provide strong constraints on this class of models.

\section{References}

Bond, J.R. \& Efstathiou. G. (1987) M.N.R.A.S., 226, pp. 655-687

Cen, R., Ostriker, J.P. \& Peebles, P.J.E (1993) Ap. J., 415, pp. 423-444

Davis, M. \& Peebles, P.J.E. (1983) Ap. J., 267. pp. 465-482

Efstathiou, G. (1988) in Large Scale Motions in the Universe: A Vatican Study Week, ed. Rubin, V.C. \& Coyne, G.V. S.J., Princeton University Press, USA.

Efstathiou. G. \& Bond. J.R. (1987) M.N.R.A.S.. 227, Short Communication, pp. 33P$38 \mathrm{P}$

Gnedin, N.Y. \& Ostriker. J.P. (1992) Ap.J., 400. pp. 1-20

Peebles, P.J.E. (1987) Nature, 327, pp. 210-211.

Schuster, J., Gaier, T.. Gundersen, J.. Meinhold. P.. Koch, T., Seiffert, M., Wuensche, C.A. \& Lubin, P. (1993) Ap. J., Vol no 412. pp. L47-L50

Udalski. A., Szymaǹski. M.. Kaluzny, J., Kubiak, M., Mateo, M. \& Krzmiǹski, W. (1994) Ap.J., Vol no 426. pp. L69-L72

Vishniac, E.T. (1987) Ap. J., 322, pp. 597-604

Walker, T.P., Steigman. G., Schramm. D.N., Olive, K.A. \& Kang, H. (1991) Ap. J., 376. pp. 51-69

Zeldovich, Ya. B. \& Sunvaev, R.B. (1970) Ap. Sp. Sc., 7, pp. 3-19 